\title{
Motion Compensation Along All Three Different Directions
}

\section{OVERVIEW}

We previously introduced the sources of variable phase in the presence of motion. In this unit, we will address how these phase perturbations can be dealt with to reduce or remove their associated artifacts. When motion causes phase dispersion across a voxel, or if the phase changes from one $T_{R}$ to the next, the bipolar gradient waveform inherent in the read or slice select gradient structures is not an ideal design. For this reason, it is necessary to redesign the gradient waveform so that both stationary and moving spins are handled appropriately. That is, the position of the spins should be encoded so that despite their motion between being excited and being measured at the echo time, they appear at the correct place in the reconstructed image and that the phase at the echo is zero for all spins. By adding new gradient lobes, this becomes possible. These new gradient waveform designs are commonly referred to as "velocity compensated."

In Figure B7.2.1A, a gradient waveform is designed to compensate the flow in the read direction. Figure B7.2.1B shows that both the stationary and moving phase terms are zero at the field echo time. (The fundamental concept of how to calculate spin phase was discussed in UNIT B7.1). To demonstrate the manifestation of motion artifacts and their

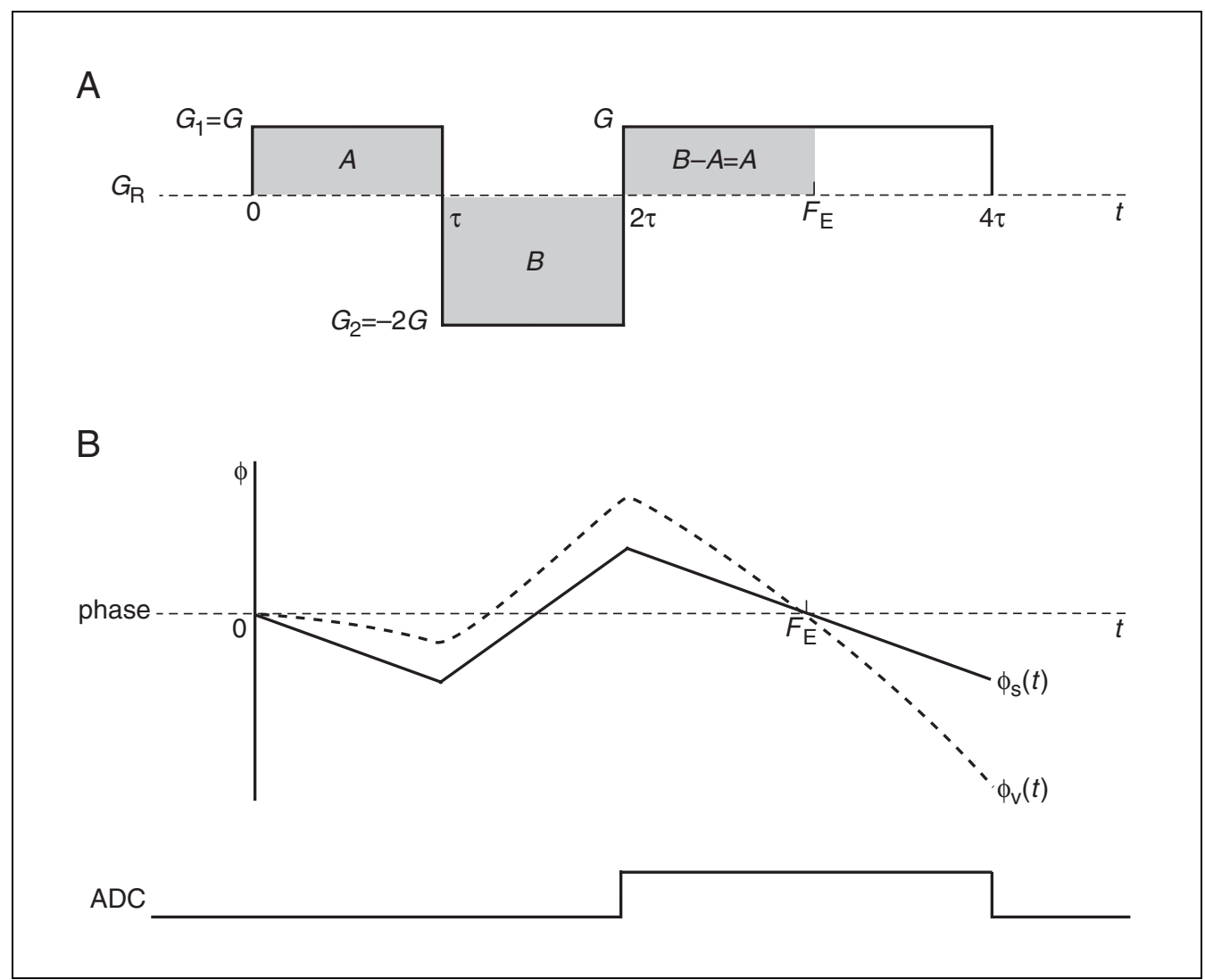

Figure B7.2.1 (A) A typical constant velocity compensated gradient waveform and (B) phase accumulated by stationary spins (solid line) and constant velocity spins (dashed line) as functions of time during the presence of such a readout gradient. The shaded gradients sum to zero as required to cause stationary spins to form an echo. The 1:-2:1 ratio of the gradient lobe areas leads to velocity compensation. The ADC on time is shown to remind the reader that sampling occurs only at the second gradient echo during the ADC on period.

Motion Artifacts, Motion Compensation and Magnetic Resonance Angiography

\section{B7.2.1}

Supplement 6 
A

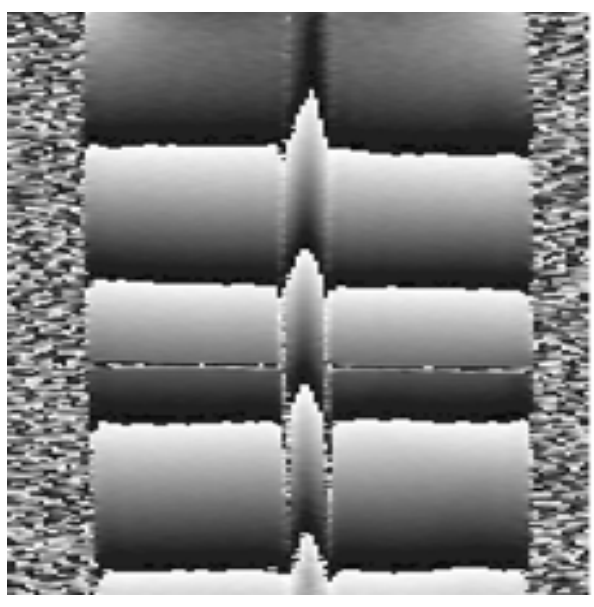

B

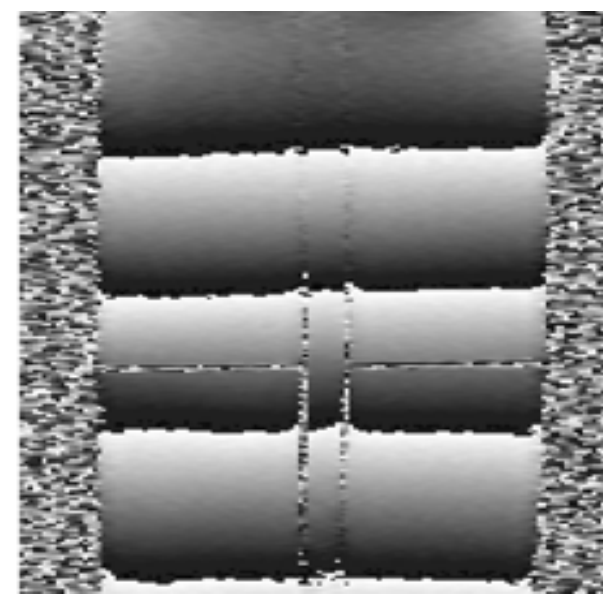

Figure B7.2.2 Phase images of a phantom containing a cylinder with flowing spins. The velocity profile of the spins in the cylinder is laminar. The cylinder is in the image plane, oriented parallel to the read direction (vertical). (A) Phase image obtained from an uncompensated read gradient structure in the presence of laminar flow demonstrates how phase is affected by velocity within the cylinder. (B) Phase image of the same object now obtained with a velocity compensated structure shows that the image phase is velocity independent. The stripes in the vertical direction represent phase changing linearly from $-\pi$ (black) to $\pi$ (bright). The four stripes in both (A) and (B) are caused purposely by a shift of echo by four sampling points $(4 \Delta t)$ in the read (vertical) direction. This feature makes it possible to view the laminar phase profile clearly. (The Fourier transform shift theorem implies that a shift in $k$-space will result in a phase variation in the reconstructed image.)

elimination, we consider several examples of uncompensated and velocity compensated images. Figure B7.2.2 shows phase images of a flow phantom before and after the compensation technique applied in the read direction. The compensated sequence successfully eliminates the phase variation across the cylinder containing the flowing spins; thus, the source of phase variation which leads to motion artifacts is gone. In Figure B7.2.3, a set of neck images is presented to compare the differences between a velocity compensated image and an uncompensated image in the read direction. The compensated image has no motion artifacts and the vessels which were originally almost completely dephased have now come back to life and are sharply delineated.

In the phase encoding direction, because the stationary phase has to satisfy the Nyquist theorem and it is not zero in general (although the phase term associated with motion has to be zero at the echo time), the design of a gradient waveform in the phase encoding direction needs some attention. An ideal design is shown in Figure B7.2.4 and a more realistic waveform is shown in Figure B7.2.5. Although it might be more involved to design a velocity compensated waveform in the phase encoding direction than in the read or slice select direction (for a 2-D sequence), one of the major concepts is that the velocity phase part is required to be zero at the echo time. When the echo time changes, the gradient waveform will need to be redesigned because the choice of time origin affects the calculation of this velocity phase term. This is discussed in the advanced material section below.

Motion Compensation Along All Three Different Directions

B7.2.2

In the slice select direction, the gradient waveform design can be as simple as in the read direction, or it can be as complicated as in the phase encoding direction if a 3-D velocity compensated image is required. Figure B7.2.6 shows the gradient waveforms in the slice select direction to null the phase (at the echo or the desired time $t_{1}$ in Fig. B7.2.6) caused 
A

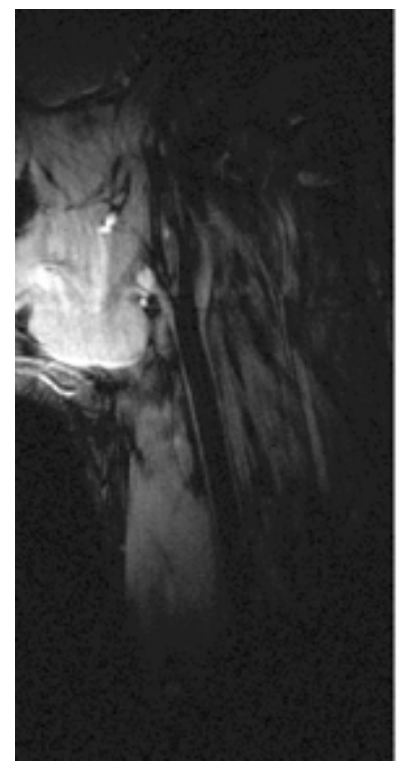

B

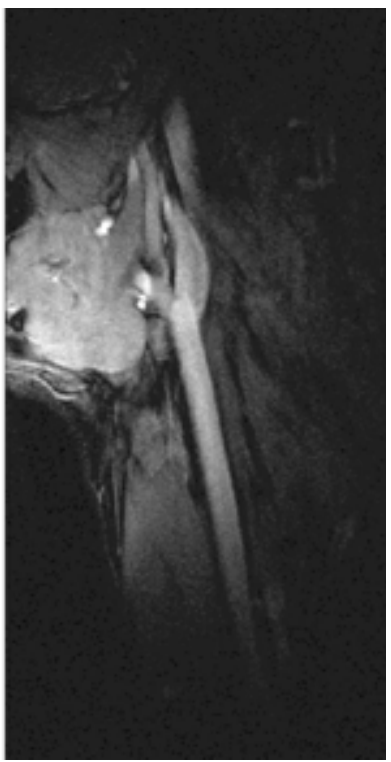

Figure B7.2.3 Images from a sagittal data set in the neck showing the importance of velocity compensation to overcome signal loss from intravoxel dephasing due to laminar flow in the read direction. (A) The intravoxel dephasing effect and consequent signal loss of the blood are well-demonstrated with an uncompensated acquisition. (B) This dephasing effect is absent in a velocity compensated image acquisition. Imaging parameters: (a) $T_{R} / T_{E}=30 \mathrm{msec} / 12 \mathrm{msec}, T_{S}=15.36$ msec, $\Delta x \times \Delta y \times \Delta z=0.39 \mathrm{~mm} \times 0.39 \mathrm{~mm} \times 3.0 \mathrm{~mm}, N_{x} \times N_{y}=512 \times 384, \theta=20^{\circ}, N_{\text {acq }}=4, \tau_{R F}=$ $1.024 \mathrm{msec}, G_{s s}=15 \mathrm{mT} / \mathrm{m}$; (b) $T_{s}=7.68 \mathrm{msec}, \tau_{\mathrm{RF}}=2.56 \mathrm{msec}, G_{s s}=8 \mathrm{mT} / \mathrm{m}$.

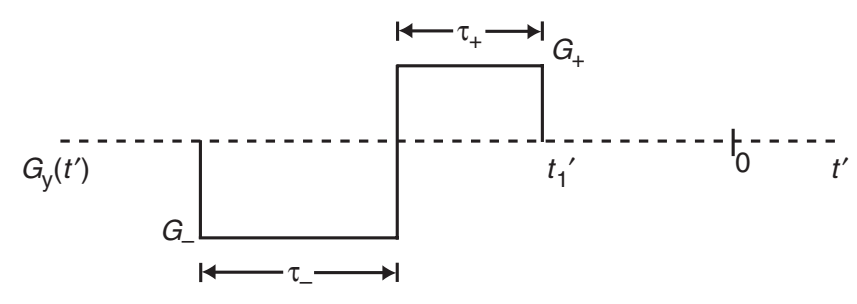

Figure B7.2.4 An ideal velocity compensated phase encoding gradient waveform.

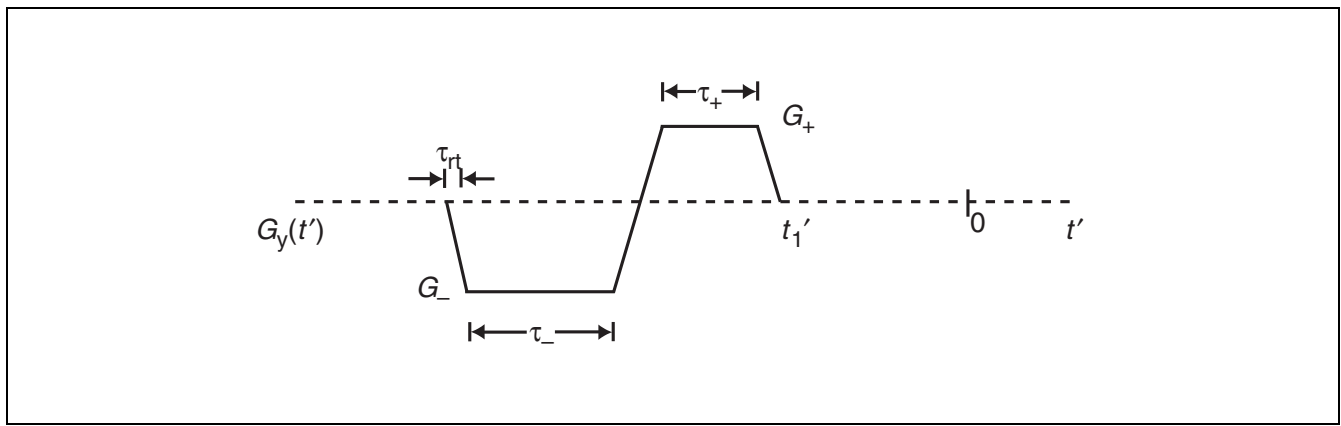

Figure B7.2.5 Velocity compensated phase encoding gradient waveform with trapezoidal lobes. All rise and fall times in the waveform are of duration $\tau_{r t}$.

Motion Artifacts, Motion Compensation and Magnetic Resonance Angiography

\section{B7.2.3}

Supplement 6 


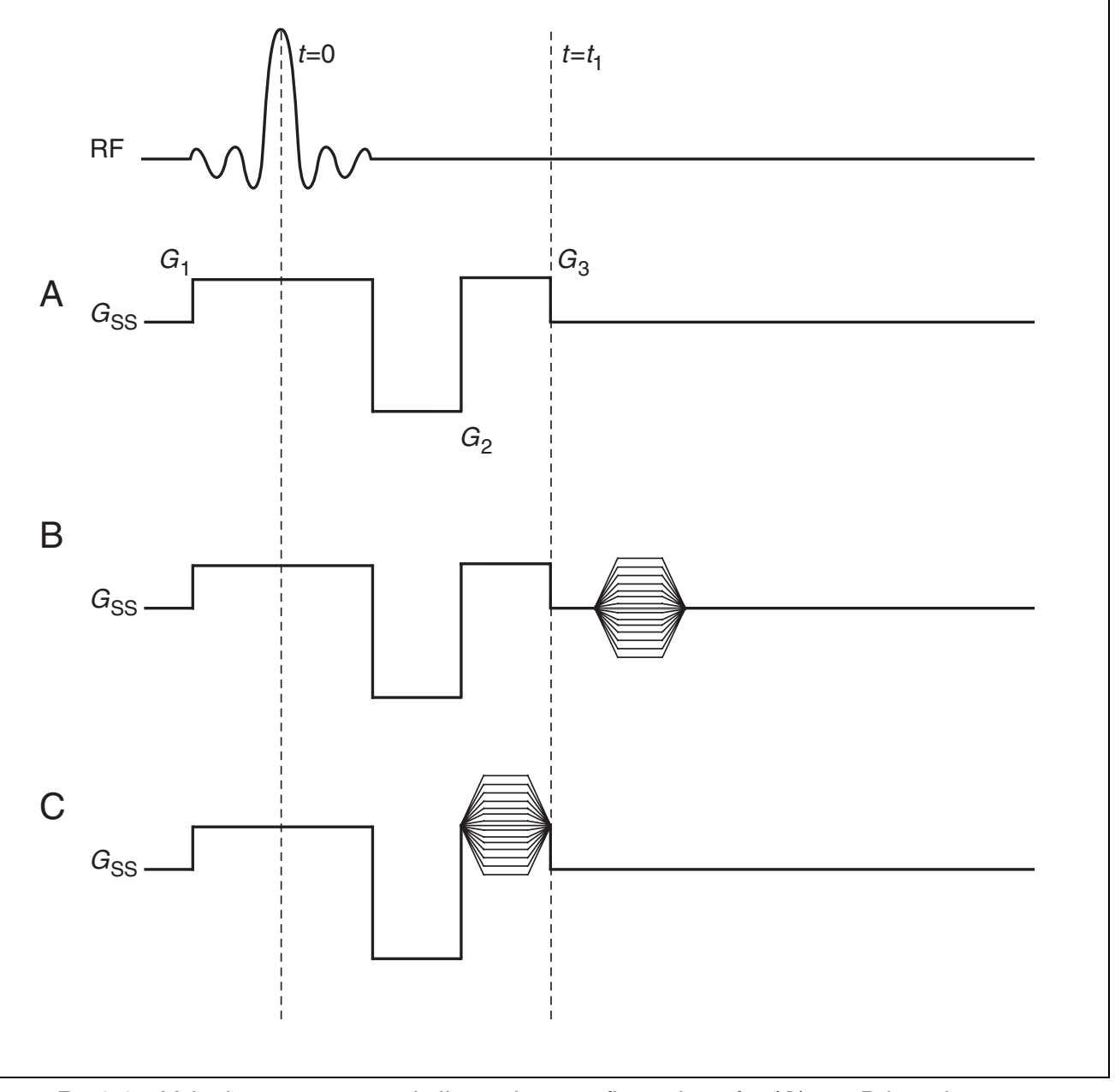

Figure B7.2.6 Velocity compensated slice select configurations for (A) a 2-D imaging sequence, (B) a 3-D imaging sequence and (C) a 3-D sequence with a shorter potential echo time. Superimposing the phase encoding table on the third lobe of the compensated slice select gradient allows a shorter $T_{E}$ but at the expense of the resolution achievable in that direction. In the 3-D examples shown in (B) and $(\mathrm{C})$, the partition encoding tables are not velocity compensated.

by flow. An example of an uncompensated and a slice select compensated transverse scan through the leg is shown in Figure B7.1.2. The image is dramatically improved with flow compensation.

Figure B7.2.7 shows a velocity-compensated sequence in all three directions (read, phase encoding, and slice select). In the slice select direction of this figure, both the slice select gradient and partition encoding tables are designed to compensate the velocity (eliminate the phase dependence on velocity, the latter specifically) at the echo time. In the advanced material section, we will discuss, in detail, how to calculate the desired compensated gradients in all three directions to obtain good images. An example of a phantom result has been shown in Figure B7.1.8. One can see that the bright line artifact (sometimes referred to as a streamline artifact) in the cylinder does not exist when the velocity compensated sequence is used.

Motion Compensation Along All Three Different Directions 

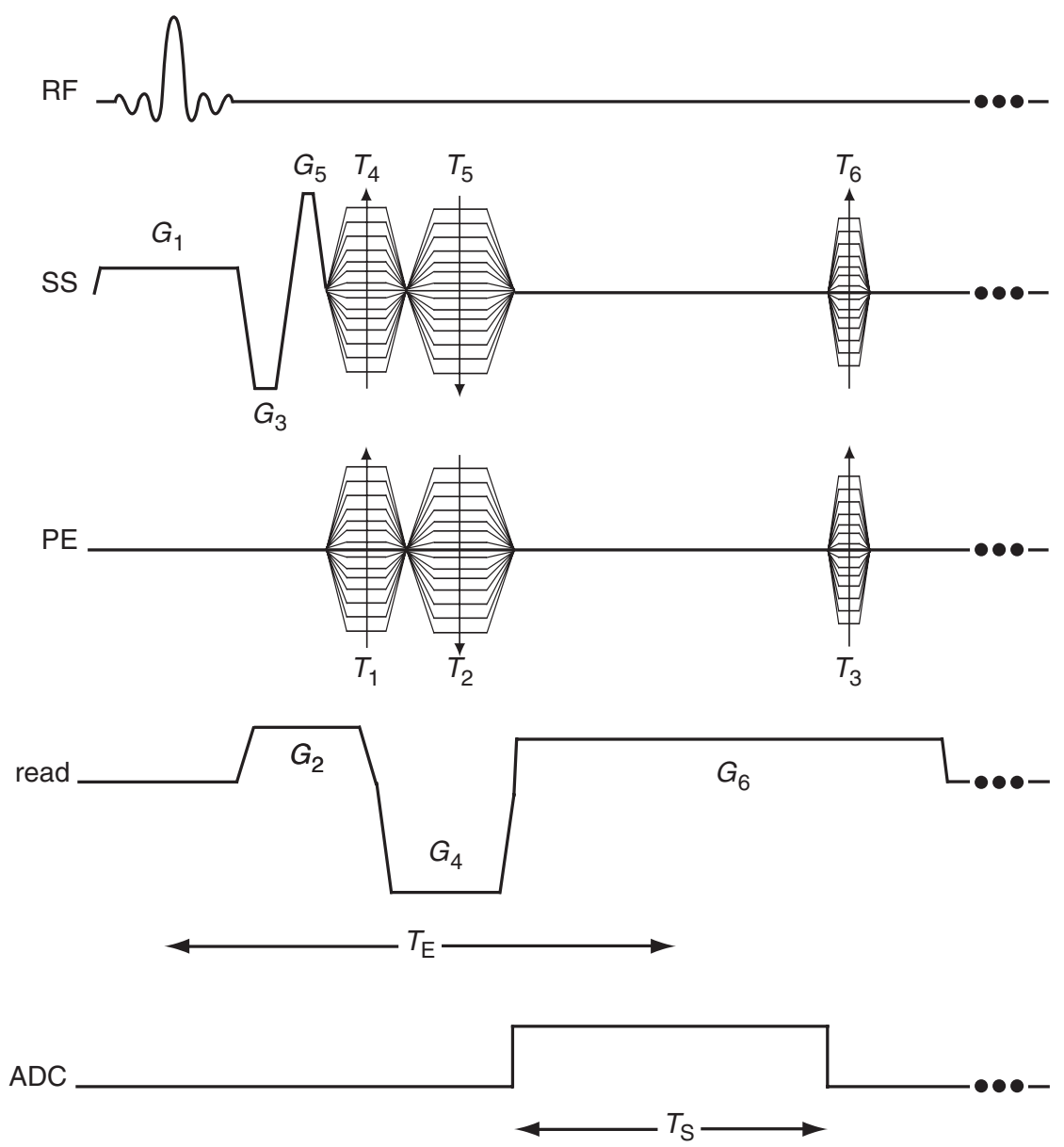

Figure B7.2.7 An actual fully velocity compensated 3-D sequence used for MR venographic imaging. All timing scales are accurately drawn from the original sequence. Gradient lobes and/or encoding tables are proportionally scaled within each direction. However, the relative magnitudes of the gradient lobes from different directions do not reflect their correct ratio in the sequence. The bandwidth of the RF pulse is $8175 \mathrm{~Hz}$ in this sequence. The echo time is $19.11 \mathrm{msec}$ and the sampling time is $12.8 \mathrm{msec}$. The starting times for the RF pulse and the first gradient lobe in the read direction are $1200 \mu \mathrm{sec}$ and $6470 \mu \mathrm{sec}$, respectively. Other sequence parameters are shown in Table B7.2.1.

\section{TECHNICAL DISCUSSION}

\section{Velocity Compensation Concepts Along the Read Direction}

One solution to the problem of image degradation due to flow along the read direction is to add another lobe to the read gradient (Fig. B7.2.1A). The additional gradient lobe is introduced to ensure that the phase at the echo is zero for both stationary and constant velocity moving spins, independent of the velocity of the moving spins. These conditions lead to two equations, one for the zeroth moment $\left(M_{0}\right)$ of $G_{R}(t)$ (which is proportional to the stationary part of the phase similar to Equation B7.1.9) and one for the first moment $\left(M_{1}\right)$ of $G_{R}(t)$ (which is proportional to the velocity portion of the phase similar to Equation B7.1.10):

\begin{tabular}{l} 
Motion Artifacts, \\
Motion \\
Compensation \\
and Magnetic \\
Resonance \\
Angiography \\
\hline
\end{tabular}

B7.2.5 
Table B7.2.1 Sequence Parameters for the Sequence Shown in Figure B7.2.7

\begin{tabular}{lccc}
\hline Label & $\begin{array}{c}\text { Ramp up/down } \\
(\mu \mathrm{sec})\end{array}$ & $\begin{array}{c}\text { Duration } \\
(\mu \mathrm{sec})\end{array}$ & $\begin{array}{c}\text { Magnitude } \\
(\mathrm{mT} / \mathrm{m})\end{array}$ \\
\hline $\mathrm{RF}$ & $\mathrm{N} / \mathrm{A}$ & 5120 & N/A \\
$G_{1}$ & 150 & 6170 & 1.5 \\
$G_{2}$ & 600 & 4430 & 2.55 \\
$G_{3}$ & 600 & 960 & -5.96 \\
$G_{4}$ & 600 & 4430 & -4.90 \\
$G_{5}$ & 600 & 300 & 5.93 \\
$G_{6}$ & 100 & 17750 & 1.83 \\
$T_{1}(\max )$ & 500 & 2210 & 9.93 \\
$T_{2}(\max )$ & 500 & 3390 & 9.93 \\
$T_{3}(\max )$ & 500 & 680 & 9.93 \\
$T_{4}(\max )$ & 500 & 2210 & 4.96 \\
$T_{5}(\max )$ & 500 & 3390 & 4.96 \\
$T_{6}(\max )$ & 500 & 680 & 4.96 \\
\hline
\end{tabular}

${ }^{a}$ The ramp down time is equal to the ramp up time in all lobes. Values in the duration column do not include either the ramp up or ramp down time. The gradient magnitude has been rounded off to the second digit after the decimal point. The following parameters are assumed in order to calculate the gradient values in the last column: $L_{x}=L_{y}=256 \mathrm{~mm}, L_{z}=128 \mathrm{~mm}, N_{x}=N_{y}=256$ and $N_{z}=64$. If $N_{x}=512$, then the magnitudes of gradients $G_{2}, G_{4}$, and $G_{6}$ are doubled in order not to change $T_{S}$.

$$
M_{0}\left(F_{E}\right)=\int_{0}^{F_{E}} G_{R}(t) d t=0
$$

and:

$$
M_{1}\left(F_{E}\right)=v_{x} \int_{0}^{F_{E}} G_{R}(t) d t=0
$$

When $v_{x}$ is zero, the phase only has a stationary component. This phase-i.e., the zeroth moment $M_{0}\left(F_{E}\right)$-must be zero to get an echo. The first moment, $M_{1}\left(F_{E}\right)$, must be zero to guarantee the velocity associated phase is zero at the field echo time $F_{E}$ independent of $v_{x}$ itself. The constant velocity $v_{x}$ is kept in Equation B7.2.2 to demonstrate that, if these two equations can be solved for a fixed set of timings, the solution will be independent of $v_{x}$. The tri-lobed design is used, generally, for velocity compensation.

Consider an example of velocity compensation. In Figure B7.2.1, the amplitudes of the two preparatory lobes $G_{1}$ and $G_{2}$ need to be found in terms of the physically limited readout gradient lobe of amplitude $G$. The two moment conditions (zeroth and first moments) lead to the following equations involving unknown gradient amplitudes and the timings shown in Figure B7.2.1:

$$
G_{1} \tau+G_{2} \tau+G \tau=0
$$

Motion

i.e.:

$$
G_{1}+G_{2}+G=0
$$

B7.2.6 
and:

$$
\frac{1}{2} G_{1} \tau^{2}+\frac{1}{2} G_{2}\left(4 \tau^{2}-\tau^{2}\right)+\frac{1}{2} G\left(9 \tau^{2}-4 \tau^{2}\right)=0
$$

or:

$$
G_{1}+3 G_{2}+5 G=0
$$

Solving these equations yields:

$$
\begin{gathered}
G_{1}=G \text { and } \\
G_{2}=-2 G
\end{gathered}
$$

This set of conditions creates a nulling of phase at the echo $\phi\left(T_{E}\right)=0$, independent of the speed of flow, known as velocity compensation (see Fig. B7.2.1). This sequence actually has two gradient echoes, one at $3 \tau / 2$ and one at $3 \tau$. (Only the second echo is flow compensated.) We discuss the concept of general even echo rephasing in the next section.

Velocity compensation avoids intravoxel spin dephasing and maintains constant phase at the echo independent of $v_{x}$. Of course, depending on the field-of-view (FOV) and resolution, it may not be possible to attain a value of $2 G$, in which case, a solution for the gradient timings must be sought to reduce the gradient amplitudes. To check this, use the Nyquist theorem (to find $G$ ) which requires:

$$
\gamma G L_{x} \Delta t=1
$$

where $L_{x}$ is the FOV in the $x$-direction and $\Delta t$ is the sampling interval along the read direction.

Generally, in designing velocity compensated sequences, it is important to minimize the echo time because of $T_{2}^{*}$ signal loss and the presence of background magnetic field gradients induced by the body itself. In addition, it is important to keep the field echo time as short as possible to avoid higher order flow effects such as those caused by acceleration or flow through such a background gradient. In any case, the application of velocity compensation can make a dramatic difference in the image quality for flowing blood, a prerequisite for good MR angiographic imaging. Such an example is shown in Figure B7.2.3.

A demonstration of how velocity compensation affects the phase of flowing spins is shown in Figure B7.2.2 where a cylinder containing flowing spins passes through an otherwise uniform phantom. The phase of the spins in Figure B7.2.2A clearly follows a parabolic (laminar) profile within the cylinder when velocity compensation is not applied. However, when velocity compensation is applied (Fig. B7.2.2B) the phase of the flowing spins is uniform and the same as that for the stationary tissue.

It is possible to add more lobes and compensate for higher-order effects such as acceleration as well. However, the acceleration-associated phase term is, usually, small compared to the stationary and velocity phase terms. It is important to remember that if motion

\begin{tabular}{l} 
Motion Artifacts, \\
Motion \\
Compensation \\
and Magnetic \\
Resonance \\
Angiography \\
\hline B7.2.7
\end{tabular}

B7.2.7 
compensation is performed up to $n$th order flow, not all higher-order effects are compensated (although some may).

\section{Even echo rephasing}

It is an interesting result that when a series of multiple gradient echoes are generated following a single RF excitation, all of the even gradient echoes may be velocity compensated. This phenomenon is referred to as even echo rephasing. For example, the structure in Figure B7.2.1A can be considered a double echo, gradient echo sequence. Sampling could take place during the negative lobe of amplitude $-2 G$ as well as during the third lobe of amplitude $G$. This is an example of even echo rephasing which in turn represents the property that a time-reversed symmetric gradient is velocity compensated. To see this, assume that $G\left(F_{E}-t\right)=G(t)$ (i.e., this is a symmetric function about the time $\left.F_{E} / 2\right)$ and that the sequence is compensated for stationary spins, i.e., the zeroth moment of $G(t)$ vanishes. This assumption leads to the following identity:

$$
\begin{aligned}
M_{0}\left(F_{E}\right) & =\int_{0}^{F_{E}} d t G(t) \\
& =\int_{0}^{F_{E} / 2} d t G(t)+\int_{F_{E} / 2}^{F_{E}} d t G\left(F_{E}-t\right) \\
& =\int_{0}^{F_{E} / 2} d t G(t)-\int_{F_{E} / 2}^{0} d u G(u) \\
& =2 \int_{0}^{F_{E} / 2} d t G(t) \\
& \equiv 0 \quad \text { (by assumption) }
\end{aligned}
$$

The time origin is set to the onset of the first gradient lobe. Then, the first moment at the field echo time where flow compensated data are acquired is:

$$
\begin{aligned}
M_{1}\left(F_{E}\right) & =\int_{0}^{F_{E}} d t t G(t) \\
& =\int_{0}^{F_{E} / 2} d t t G(t)+\int_{F_{E} / 2}^{F_{E}} d t t G\left(F_{E}-t\right) \\
& =\int_{0}^{F_{E} / 2} d t t G(t)-\int_{F_{E} / 2}^{0} d u\left(F_{E}-u\right) G(u) \\
& =\int_{0}^{F_{E} / 2} d t t G(t)-\int_{0}^{F_{E} / 2} d u u G(u)+F_{E} \int_{0}^{F_{E} / 2} d u G(u) \\
& =F_{E} \int_{0}^{F_{E} / 2} d u G(u)
\end{aligned}
$$

Because the first echo occurs at $F_{E} / 2$, that is, the zeroth moment is zero there, $M_{1}\left(F_{E}\right)=$ 0 as postulated.

\section{Importance of time-origin in moment calculations}

Suppose there is a general gradient waveform $G(t)$ with no special properties and that we want to calculate the $n$th moment of this gradient waveform at time $t_{1}$. Assume that the gradient waveform starts at time $t_{0}$ and is zero prior to this time. Then:

$$
M_{n}\left(t_{0}, t_{1}\right)=\int_{t_{0}}^{t_{1}} d t t^{n} G(t)
$$

by definition. Shifting origins so that $t^{\prime}=t-t_{0}$ : 


$$
M_{n}\left(t_{0}, t_{1}\right)=\int_{t_{0}}^{t_{1}-t_{0}} d t^{\prime}\left(t^{\prime}+t_{0}\right)^{n} G\left(t^{\prime}+t_{0}\right)
$$

Expanding $\left(t^{\prime}+t_{0}\right)^{n}$ in a binomial expansion gives:

$$
\begin{aligned}
M_{n}\left(t_{0}, t_{1}\right) & =\int_{t_{0}}^{t_{1}} d t t^{n} G(t) \\
& =\int_{0}^{t_{1}-t_{0}} d t^{\prime}\left(\left(t^{\prime}\right)^{n}+\left(\begin{array}{l}
n \\
1
\end{array}\right) t_{0}\left(t^{\prime}\right)^{n-1}+\left(\begin{array}{l}
n \\
2
\end{array}\right) t_{0}^{2}\left(t^{\prime}\right)^{n-2}+\ldots+t_{0}^{n}\right) G\left(t^{\prime}+t_{0}\right)
\end{aligned}
$$

From Equation B7.2.14, it is clear that $M_{n}\left(t_{0}, t_{1}\right)$ is the same as $M_{n}\left(0, t_{1}-t_{0}\right)$, i.e., the $n$th moment is independent of time-origin, if and only if:

$$
\begin{aligned}
\int_{0}^{t_{1}-t_{0}} d t^{\prime}\left(t^{\prime}\right)^{n-1} G\left(t^{\prime}+t_{0}\right) & =\int_{0}^{t_{1}-t_{0}} d t^{\prime}\left(t^{\prime}\right)^{n-2} G\left(t^{\prime}+t_{0}\right)=\ldots \\
& =\int_{0}^{t_{1}-t_{0}} d t^{\prime} G\left(t^{\prime}+t_{0}\right)=0
\end{aligned}
$$

In conclusion, the time-origin in a general nth moment calculation can be chosen arbitrarily only when all lower-order moments of that gradient waveform are zero at the point of interest; otherwise, moment calculations must be with respect to a particular time (for example, $T_{E}$ ). This will have important consequences for studying velocity compensation for the phase encoding gradient.

\section{Velocity Compensation Along the Slice Select Direction}

Flow or motion which occurs along the slice select direction during the slice select process can also lead to signal loss and image degradation. Velocity compensation in the slice select direction is critical to obtaining an artifact-free image in the presence of motion (see Fig. B7.1.2). Motion compensation along the slice select direction is achieved by adding an additional slice select gradient lobe, using an analogous method to that employed in the read gradient case. Gradient lobes are chosen such that the zeroth and first moment of the slice select gradients are zero at the end of the slice select process $(t$ $=t_{1}$ ) as demonstrated in Figure B7.2.6. The approximation is generally used that the center of the RF pulse is the time when spins are tipped into the transverse plane $(t=0)$ which is accurate for low flip angles, at least. To demonstrate the design, the analogous structure from Figure B7.2.1 is shown in Figure B7.2.6 for the slice select direction in a 2-D (Fig. B7.2.6A) and a 3-D (Fig. B7.2.6B) sequence.

In the real 3-D sequence shown in Figure B7.2.7, the slice select gradients compensate the flow at the echo time as well as at the end of these gradient lobes (at the starting time of the partition encoding tables). This is because the time-origin of the first moment does not matter (as discussed above) when the zeroth moment is zero. In this case, when these gradient lobes eliminate the phase caused by flow, both the zeroth and first moments are zero at any time after the end of these gradient lobes. The time-origin becomes important when we place the encoding tables in the sequence where the sum of zeroth moments is not zero.

Note that the phase encoding in a 3-D sequence can be done separately if a short $T_{E}$ is not required (Fig. B7.2.6B). This also allows a higher resolution in the slice select direction. For example, when the partition encoding is superimposed on top of gradient $G_{3}$ (Fig. B7.2.6C), the Nyquist criterion limits the resolution in the $z$ direction to be:

Motion Artifacts,
Motion
Compensation
and Magnetic
Resonance
Angiography
B7.2.9

Supplement 6 


$$
\Delta z^{\prime}=\frac{1}{\psi\left(G_{\max }-G_{3}\right) \tau_{p e}}
$$

where $G_{\max }$ is the maximum gradient set by the hardware limitation and $\tau_{p e}$ is the duration of the encoding. For the same number of partition encoding steps, clearly, if $G_{3}$ is zero, i.e., if the encoding table is placed separate from $G_{3}$, then the highest resolution in the $z$ direction which can be achieved is:

$$
\Delta z=\frac{1}{\gamma G_{\max } \tau_{p e}}
$$

On the other hand, combining the third lobe with the phase encoding table allows the echo time to be shortened (Fig. B7.2.6C).

\section{Velocity Compensation Along Phase Encoding Direction}

In the phase encoding direction, say $y$ (as well as for the partition encoding direction for 3-D imaging), the effects of motion are different from those in the read direction. For this section, constant plug-like flow will be assumed since the results obtained here can be extended easily to the more general laminar flow and pulsatile flow cases. The vessel is also assumed to be in-plane so there is no flow along the slice select direction.

\section{Velocity compensating phase encoding gradients}

As shown in the previous unit, the primary motion effect in the phase encoding direction is a spatial shift of the vessel which occurs because of movement of the isochromat between times $t_{p e}$ and $T_{E}$ (see Fig. B7.1.7). The solution to the shift artifact lies in designing a phase encoding gradient which is capable of encoding the position of the constant velocity isochromat at the echo rather than at the center of the phase encoding table (as it occurs in the single phase encoding gradient lobe case). In the signal processing view of this problem, the spatial misregistration was seen to be a manifestation of the extra phase accumulated by moving isochromats because the single-lobed gradient had a nonzero first moment. Therefore, a velocity compensating phase encoding gradient is needed to null the first moment on a time-scale with the echo time as its origin, even though the zeroth moment is not nulled here as it is in the case of a velocity compensating read gradient. Mathematically stated, the two equations which need to be satisfied by a velocity compensating phase encoding gradient waveform are:

$$
M_{0}(0) \equiv \int_{-\infty}^{0} \Delta G_{y}(t) d t=\frac{1}{\psi L_{y}}
$$

and

$$
M_{1}(0) \equiv \int_{-\infty}^{0} t \Delta G_{y}(t) d t=0
$$

where the time origin is at the echo time $T_{E}$. From a physical perspective, when the data are read out using a read gradient, all spins at a given $x$ position (at the echo time $T_{E}$ ) will be measured. They will have come from somewhere else but are all measured at one $x$ value. Therefore, solving for Equation B7.2.18 and Equation B7.2.19 will prevent the usual misregistration artifact but only with respect to the time $T_{E}$.

Using the experience acquired in velocity compensating the read gradient, consider two pulsed phase encoding gradients, where a second lobe is added to give an additional degree of freedom so that the first moment could be nulled at the echo time as well as satisfying the zeroth moment requirement for phase encoding. The velocity compensated gradient waveform is shown in Figure B7.2.4 for a single phase encoding step. The zeroth moment condition reduces to:

\section{B7.2.10}




$$
G_{+} \tau_{+}+G_{-} \tau_{-}=\frac{1}{\psi L_{y}}
$$

and the first moment condition reduces to:

$$
\frac{G_{+} \tau_{+}}{2}\left(2 t_{1}^{\prime}-\tau_{+}\right)+\frac{G_{-} \tau_{-}}{2}\left(2\left(t_{1}^{\prime}-\tau_{+}\right)-\tau_{-}\right)=0
$$

Substituting Equation B7.2.20 into Equation B7.2.21 gives:

$$
G_{-}\left(\tau_{+} \tau_{-}+\tau_{-}^{2}\right)=\frac{2 t_{1}^{\prime}-\tau_{+}}{\psi L_{y}}
$$

and finally:

$$
G_{-}=\frac{1}{\gamma L_{y} \tau_{-}} \frac{\left(2 t_{1}^{\prime}-\tau_{+}\right)}{\left(\tau_{-}+\tau_{+}\right)}
$$

Then, $G_{+}$is given by:

$$
G_{+}=\frac{1}{\tau_{+}}\left(\frac{1}{\psi L_{y}}-G_{-} \tau_{-}\right)=\frac{-2 t_{1}^{\prime}+2 \tau_{+}+\tau_{-}}{\psi L_{y} \tau_{+}\left(\tau_{+}+\tau_{-}\right)}
$$

By identically (and appropriately) scaling both $G_{+}$and $G_{-}$based on the Nyquist criterion, the usual phase encoding is accomplished. The $T_{E}$ dependence of the velocity compensating phase encoding gradient waveform means that, in a multi-echo sequence, the velocity compensation conditions will be satisfied only for the first echo unless the phase encoding table is modified between echoes.

Taking a closer look at the problem at hand, there are two equations that must be satisfied; yet, in the above-described procedure, there are four freely variable parameters $\left(G_{-}, G_{+}\right.$, $\tau_{-}$, and $\left.\tau_{+}\right)$. This means that there are more degrees of freedom than equations. As a result, this system of equations leads to more than one possible solution. If more constraints are included in the gradient waveform design stage, it may be possible to obtain a unique solution by solving a constrained, nonlinear optimization problem as long as the constraints are reasonable. Possible constraints on $t_{1}^{\prime}$ come from field echo time in the readout direction and spatial resolution in the phase encoding direction. The gradient-step waveform thus obtained, and the maximum gradient capability of the system will then determine the maximum number of phase encoding steps $\left(N_{y, \max }\right)$ and, hence, the spatial resolution along $y$.

A more realistic gradient waveform along the phase encoding direction is shown in Figure B7.2.5. We can follow the same procedure presented above to obtain the appropriate values of $G_{+}$and $G_{-}$such that this waveform is velocity compensated. Again, the zeroth moment should satisfy:

$$
G_{-}\left(\tau_{-}+\tau_{R F}\right)+G_{+}\left(\tau_{+}+\tau_{R F}\right)=\frac{1}{\not L_{y}}
$$

Motion Artifacts, Motion Compensation and Magnetic Resonance Angiography

B7.2.11 
and the first moment should satisfy:

$$
G_{-}\left(\tau_{-}+\tau_{R F}\right)\left(2 t_{1}^{\prime}-6 \tau_{R F}-2 \tau_{+}-\tau_{-}\right)+G_{+}\left(\tau_{+}+\tau_{R F}\right)\left(2 t_{1}^{\prime}-2 \tau_{R F}-\tau_{+}\right)=0
$$

These two equations give the required values of $G_{+}$and $G_{-}$to compensate velocity in the phase encoding direction.

$$
\begin{aligned}
& G_{+}=\frac{2 \tau_{+}+\tau_{-}+6 \tau_{R F}-2 t_{1}^{\prime}}{\not L_{y}\left(\tau_{+}+\tau_{R F}\right)\left(\tau_{+}+\tau_{-}+4 \tau_{R F}\right)} \\
& G_{-}=\frac{2 t_{1}^{\prime}-2 \tau_{R F}-\tau_{+}}{\not L_{y}\left(\tau_{-}+\tau_{R F}\right)\left(\tau_{+}+\tau_{-}+4 \tau_{R F}\right)}
\end{aligned}
$$

A comparison of a compensated and an uncompensated sequence in the phase encoding direction is shown in Figure B7.1.8. The laminar profile generates a marked peak to the left since the rapid center flow shifts a large distance (in this instance, to the edge of the tube) while the spins to the left of the center shift less but still tend to fall close to the left edge. The sum total of these shifts gives the profile shown in Figure B7.1.8C. The velocity compensated sequence does an excellent job of putting the spins back to where they belong (as evident in the rather uniform profile across the tube in Fig. B7.1.8D).

\section{ACKNOWLEDGEMENTS}

The authors would like to thank Jan Koonce for her secretarial work.

\section{KEY REFERENCES}

Axel, L. and Morton, D. 1987. MR flow imaging of velocity compensated/uncompensated difference images. J. Comput. Assist. Tomogr. 11:31.

This paper describes the concepts involved in velocity compensation.

Bradley, W.G. 1988. Flow phenomena in MR imaging. Am. J. Roentgenol. 150:983.

The concept of even echo rephasing is described in this article.

Frank, L.R., Crawley, A.P., and Buxton, R.B. 1992. Elimination of oblique flow artifacts in magnetic resonance imaging. Magn. Reson. Med. 25:299.

The concept of velocity compensation along the phase encoding direction is described in this paper.

Haacke, E.M. and Lenz, G.W. 1987. Improving MR image quality in the presence of motion by using rephasing gradients. Am. J. Roentgenol. 148:1251.

This paper describes the concepts involved in velocity compensation.

Haacke, E.M., Brown, R.W., Thompson, M.R., and Venkatesan, R. 1999. Magnetic Resonance Imaging: Physical Principles and Sequence Design. John Wiley \& Sons, New York.

This text covers the technical aspects presented here, but in more detail, and also discusses more advanced materials.

Nayler, G.L., Firmin, D.N., and Longmore, D.B. 1986. Blood flow imaging by cine magnetic resonance. J. Comput. Assist. Tomogr. 10:715.

This paper describes the concepts involved in velocity compensation.

Nishimura, D.G., Macovski, A., and Pauly, J.M. 1986. Magnetic resonance angiography. IEEE Trans. Med. Imaging MI-5:140.

This paper describes the concepts involved in velocity compensation. 
Contributed by Yu-Chung Norman Cheng

Case Western Reserve University

Cleveland, Ohio

E. Mark Haacke

The MRI Institute for Biomedical Research

Detroit, Michigan

Motion Artifacts,

Motion

Compensation

and Magnetic

Resonance

Angiography

B7.2.13 